\title{
Utilidad de los hemocultivos en el tratamiento antimicrobiano de la neumonía neumocóccica bacteriémica en el adulto
}

\author{
Sebastián Mathurin L., Andrés Agüero L., Celia Jaimet A., \\ Erica Schälibaum G., Mariela Demergasso S. y Victoria Acuña S.
}

\section{Utility of blood cultures on antibiotic therapy in adult bacteremic pneumococcal pneumonia}

We evaluated the utility of blood cultures in the therapeutic management of patients with bacteremic pneumococcal pneumonia admitted to an internal medicine unit, in a retrospective observational study. Forty-nine patients were included, $75.5 \%$ were men, mean age 51.1 years. All S. pneumoniae strains were susceptible to penicillin. Four patients died. In $75.5 \%$ the treatment was modified narrowing antibiotic spectrum, in $57 \%$ cases it was changed to bencylpenicillin or amoxicillin exclusively, but only in $16 \%$ within the first 4 days. In 12 cases the prescription coincided with the oral switch therapy to amoxicillin. Due to the benefits and potential advantages of penicillin in diminishing the incidence of antibiotic resistance and reducing costs, it is important to work on prescription habits among physicians. This is especially important in the case of penicillin and the opportune moment of therapy change, improving the use of the microbiological report.

Key words: Blood cultures, Streptococcus pneumoniae, pneumococcal pneumonia, bacteremia, microbiology, antibiotics.

Palabras clave: Hemocultivos, Streptococcus pneumoniae, bacteriemia, microbiología, antimicrobianos, neumonía neumocóccica.

\section{Introducción}

$\mathrm{L}$ os hemocultivos se realizan en la mayoría de los pacientes con neumonía adquirida en la comunidad (NAC) que se internan, para identificar el agente etiológico y es una recomendación de las guías de práctica clínica para el manejo de la $\mathrm{NAC}^{1-6}$. Uno de los principales objetivos es detectar microorganismos resistentes al tratamiento empírico inicial. Sin embargo, actualmente se indican tratamientos antimicrobianos de amplio espectro, por lo que la principal utilidad se limita a estimular la reducción del espectro del tratamiento antibacteriano inicial. La relación costo-efectividad de los hemocultivos de rutina para el manejo de la NAC ha sido cuestionada en diferentes publicaciones, por la baja frecuencia de resultados positivos y el reducido impacto en modificar el tratamiento empírico inicial ${ }^{7-9}$.

Streptococcus pneumoniae es el principal agente causal de NAC. La presentación con bacteriemia es más frecuente que en otras causas de neumonía, observándose entre 6 y $46 \%$ de todos los casos de neumonía neumocóccica ${ }^{10-11}$ y está asociada con una mortalidad tres veces mayor que la forma no bacteriémica. Presentan una mayor incidencia de infecciones neumo- cóccicas invasoras los pacientes sobre 65 años de edad y aquellos con co-morbilidades ${ }^{12}$.

La aparición de cepas de S. pneumoniae resistentes a bencil-penicilina (de ahora en adelante "penicilina") en los últimos años es un problema en algunas regiones del mundo donde se reportan cifras de resistencia global mayores al $30 \%{ }^{13}$. En las neumonías, las cepas de S. pneumoniae con sensibilidad disminuida a penicilina (SDP) no se han relacionado con fracasos terapéutico ni aumento de la mortalidad ${ }^{14-16}$. Debido a la alta concentración que alcanzan en el pulmón los antimicrobianos utilizados para el tratamiento de la NAC, el Drug-Resistant Streptococcus pneumoniae Therapeutic Working Group (DRSPWG) ha propuesto como punto de corte en neumonía los siguientes valores de CIM para penicilina: susceptible con CIM $\leq 1$ $\mu \mathrm{g} / \mathrm{ml}$, resistencia intermedia (RI) con CIM de $2 \mu \mathrm{g} / \mathrm{ml}$ y resistencia alta (RA) con CIM $\geq 4 \mu \mathrm{g} / \mathrm{ml}$, quedando limitada la posibilidad de fracaso terapéutico a aquellas infecciones causadas por cepas con CIM $\geq 4 \mu \mathrm{g} /$ $\mathrm{ml}^{17}$. De esta manera, el aislado de $S$. pneumoniae en los hemocultivos de pacientes internados con NAC permitiría, en la mayoría de los casos, reducir el espectro antibacteriano y los costos utilizando penicilina, ampicilina y/o amoxicilina, esta última como opción
Rosario, Santa Fe, Argentina Hospital Intendente Carrasco Servicio de Clínica Médica

Recibido: 25 de marzo 2008 Aceptado: 28 de noviembre 2008

Conflicto de intereses: Los autores declaran no tener conflictos de intereses

Financiamiento: El trabajo se realizó sin aportes económicos o becas.

Correspondencia a: Andrés Agüero L. andresaguero@argentina.com 
para la vía oral. Existen reportes que estudian la conducta médica, cuando en los pacientes con neumonía se aísla $S$. pneumoniae, que revelan una baja prescripción de penicilina ${ }^{8,9}$.

El objetivo del presente trabajo retrospectivo es evaluar la utilidad e impacto de los resultados de los hemocultivos en el manejo terapéutico de los pacientes adultos con neumonía neumocóccica bacteriémica en nuestro hospital.

\section{Pacientes y Métodos}

Pacientes. Estudio observacional, retrospectivo. Se incluyeron aquellos pacientes sobre 14 años de edad, con NAC y al menos un hemocultivo positivo para $S$. pneumoniae, internados en forma consecutiva en el Servicio de Clínica Médica del Hospital Intendente Carrasco, desde enero del 2005 a diciembre del 2007. El hospital es municipal, de segundo nivel y en él se atienden predominantemente pacientes de la zona oeste de la ciudad de Rosario. Se utilizó el estudio consecutivo de los pacientes internados para evitar cualquier sesgo en la selección de pacientes. Se excluyeron aquellos pacientes con una estadía hospitalaria menor de 48 horas.

Métodos. Se revisaron las historias clínicas de los pacientes con diagnóstico de NAC con bacteriemia por $S$. pneumoniae, y se recogieron los datos siguiendo un protocolo previamente diseñado que incluía variables epidemiológicas (edad, sexo, mes de diagnóstico, enfermedades predisponentes, consumo de alcohol y tabaco, internaciones previas en el último año, antibioterapia en los últimos tres meses, neumonías previas, días de internación), manifestaciones clínicas (fiebre, tos, expectoración, disnea, dolor torácico, calofríos, cefalea, dolor abdominal, auscultación patológica, vómitos, diarrea, confusión mental, oliguria, shock, signos vitales), características de laboratorio (hemograma, gasometría arterial, uremia, creatininemia, natremia, glicemia, albuminemia e imágenes radiológicas), índice de gravedad de neumonía (ISN) de acuerdo con el protocolo de Fine y col $^{18}$, informe microbiológico (susceptibilidad in vitro de la cepa a penicilina), tratamiento específico (empírico inicial, cambio de antimicrobiano y motivos del mismo, duración) y variables evolutivas (alta, complicaciones, fallecimiento).

Criterios para el diagnóstico de infección: Las neumonías fueron confirmadas con criterios clínicos (tos, expectoración, fiebre, disnea, dolor torácico, alteración del estado mental, semiología respiratoria de consolidación), de laboratorio (leucocitosis) y radiológicos (nuevo infiltrado no explicado por otra causa).
Fue registrada la presencia de otros microorganismos en sangre.

Definiciones. Infección adquirida en la comunidad: síntomas respiratorios aparecidos o cultivos positivos obtenidos en las primeras 48 horas de internación más la ausencia de internación en los 15 días previos. Extensión radiográfica, lobar o multilobar (pudiendo ser el compromiso de más de un lóbulo unilateral o bilateral), y bilateral: opacidades difusas con patrón reticular, reticulonodulillar o nodulillar. Se registró la presencia de efusión pleural. Shock: presencia de una presión arterial sistólica menor a 90 $\mathrm{mmHg}$, refractaria al aporte de fluidos y/o anormalidades de la perfusión tisular con una excreción urinaria inferior a $40 \mathrm{ml} /$ hora, que requiere el uso de agentes vasopresores. Complicaciones, cualquiera de las siguientes condiciones: presencia de efusión pleural o empiema que requiera la colocación de tubo de tórax, ingreso a unidad de terapia intensiva (UTI), asistencia con ventilación mecánica (VM), neumonía de lenta resolución y aquellas otras que pudieran alterar la evolución y la respuesta al tratamiento adecuado. Mortalidad: fallecimiento del paciente con NAC y hemocultivos positivos para $S$. pneumoniae durante la internación, sin que fuera necesariamente consecuencia directa de la infección neumocóccica.

Análisis microbiológico. Los hemocultivos se extrajeron y procesaron según las técnicas, métodos y recomendaciones al uso, utilizando botellas con medio líquido y lectura fluorométrica en el sistema BACTEC ${ }^{\circledR}$. Los aislados e identificaciones se llevaron a cabo de acuerdo con los métodos habituales en microbiología clínica: mediante la morfología típica de la bacteria en la tinción de Gram (cocos grampositivos, lanceolados, agrupados en pares o cadenas cortas), prueba de catalasa negativa, susceptibilidad a optoquina y prueba de solubilidad en bilis positiva, características típicas de crecimiento de las colonias en placas de MüllerHinton suplementadas con $5 \%$ de sangre de cordero. La susceptibilidad a penicilina se estudió mediante el tamizaje con discos de $1 \mu$ de oxacilina siguiendo los criterios del Clinical and Laboratory Standards Institute (CLSI). Se determinó la CIM para penicilina mediante epsilometría $\left(\mathrm{E}^{- \text {test }^{\mathbb{R}}}\right)^{19}$.

Tratamiento anti-infeccioso. Se consideró cambio del tratamiento empírico inicial si se suspendió o agregó uno o más antimicrobianos. El cambio a vía oral con un antibacteriano de similar espectro al utilizado por vía parenteral no fue considerado un cambio de tratamiento. Se consideró reducción de espectro si se suspendió la cobertura para microorganismos atípicos (incluido el tratamiento con cotrimoxazol para Pneumocystis jirovecii, indicado en pacientes con infección por VIH como una parte del tratamiento empírico 
inicial) y/o se cambió el tratamiento con ceftriaxona o ampicilina/sulbactam por penicilina, ampicilina o amoxicilina.

Se definió como: utilización óptima del hemocultivo si el tratamiento se cambió a penicilina, ampicilina y/o amoxicilina, como único antibacteriano, con suspensión de la cobertura para microorganismos atípicos o anaerobios si se hubiera indicado como parte del tratamiento empírico inicial; utilización oportuna si el cambio se realizó dentro de los primeros cuatro días y utilización tardía si el cambio se realizó a partir del quinto día de internación.

El protocolo de investigación fue aprobado por el Comité de Docencia e Investigación del hospital. No fue necesario el consentimiento informado de cada paciente porque fue un estudio retrospectivo sin intervención del investigador. Los datos personales obtenidos de los pacientes se consideraron secreto médico.

Análisis estadístico. Las variables continuas están expresadas como promedio \pm DS y las variables categóricas como porcentajes. Las técnicas estadísticas utilizadas fueron el test de $\chi^{2}$ con corrección de Yates para estudiar asociación entre variables categóricas y en caso de menos de cinco individuos esperados con prueba exacta de Fisher, y la prueba t de student para variables continuas con distribución paramétrica. En el caso de variables con distribución no paramétrica fueron realizados con el test de Mann-Whitney/ Wilconxon. El nivel de significación considerado para el análisis estadístico fue de $5 \%$. Los datos fueron analizados con el paquete estadístico Epi-Info 2002 versión 3.3.2 (CDC, Atlanta).

\section{Resultados}

Fueron identificados en los informes microbiológicos de los pacientes internados en la sala de clínica médica 60 episodios de bacteriemia por S. pneumoniae, de los cuales 53 tuvieron un origen pulmonar, cuatro con neumonías intra-hospitalarias y 49 con NAC; estos 49 pacientes hospitalizados en forma consecutiva con NAC y bacteriemia por S. pneumoniae fueron el grupo estudiado. Se incluyeron $37(75,5 \%)$ hombres y $12(24,5 \%)$ mujeres. La edad media de los pacientes ( \pm DS) fue de 51,1 $\pm 17,2$ años (rango 15-79 años). El $65 \%$ se diagnosticó en otoño/invierno. Los pacientes bajo 40 años de edad eran $16(33 \%)$ de los cuales seis tenían infección por VIH y los pacientes sobre 65 años fueron $13(26,5 \%)$. Tuvieron internación previa en el último año ocho casos (16\%) y antecedente de neumonía 12 pacientes $(24,5 \%)$. Diez por ciento recibió antibioterapia dentro de los tres meses previos a su ingreso hospitalario.
Los datos clínicos más frecuentemente encontrados fueron auscultación patológica $(96 \%)$, tos (92\%), fiebre $(88 \%)$ y expectoración $(82 \%)$. Tuvieron una frecuencia cardíaca $\geq 100 / \min 59 \%$, una frecuencia respiratoria $\geq 30 / \min 35 \%$ y presión arterial sistólica $\leq 90$ $\mathrm{mmHg} 29 \%$.

En 12 pacientes $(24,5 \%)$ el infiltrado radiológico fue multilobar, en 35 (71,5\%) fue lobar, tuvieron un compromiso bilateral difuso $2(4 \%)$ y se detectó efusión pleural en siete pacientes $(14,6 \%)$.

Ochenta y ocho por ciento presentó algún hábito o co-morbilidad predisponentes y $61 \%$ tenía dos o más factores predisponentes, siendo más frecuentes el tabaquismo, el alcoholismo, la hepatopatía crónica, la diabetes mellitus, la enfermedad pulmonar obstructiva crónica (EPOC) y la infección por VIH (Tabla 1).

Se hizo el cálculo del ISN con los siguientes resultados: grupo I, tres casos; grupo II, nueve casos; grupo III, 10 casos; grupo IV, 21 casos y grupo V, seis casos. En el grupo de bajo riesgo (grupos I, II y III) hubo un total de 22 casos $(45 \%)$ y en el grupo de moderado a alto riesgo (grupos IV y V) hubo 27 casos (55\%).

Tabla 1. Factores predisponentes y co-morbilidades en pacientes adultos con neumonía neumocóccica bacteriémica

\begin{tabular}{|c|c|c|}
\hline \multirow{2}{*}{ Factor predisponerte y/o co-morbilidad } & \multicolumn{2}{|c|}{ Total n: 49} \\
\hline & $\mathbf{n}$ & $(\%)$ \\
\hline Alcoholismo (> 80 g/día) & 19 & (39) \\
\hline Tabaquismo activo o ex-fumador $<2$ años & 23 & $(47)$ \\
\hline Adicción a drogas & 7 & $(14)$ \\
\hline Hepatopatía crónica - cirrosis hepática & 9 & $(18)$ \\
\hline Diabetes mellitus & 8 & $(16)$ \\
\hline EPOC & 7 & $(14)$ \\
\hline Infección por VIH & 7 & $(14)$ \\
\hline Insuficiencia renal crónica & 2 & (4) \\
\hline Neoplasia & 1 & $(2)$ \\
\hline Insuficiencia cardíaca o cardiopatía crónica & 1 & $(2)$ \\
\hline Enfermedad del tejido conectivo & 1 & $(2)$ \\
\hline Otras* & 6 & $(16)$ \\
\hline \multicolumn{3}{|l|}{ Número de factores predisponentes: } \\
\hline 1 & 13 & $(27)$ \\
\hline 2 & 18 & $(37)$ \\
\hline 3 & 6 & $(12)$ \\
\hline 4 & 5 & $(10)$ \\
\hline 5 & 1 & $(2)$ \\
\hline Sin factor predisponerte & 6 & $(12)$ \\
\hline
\end{tabular}


El estudio de susceptibilidad in vitro se realizó en todas las muestras con un solo caso de susceptibilidad disminuida a penicilina, con resistencia intermedia de acuerdo con el criterio poblacional del $\mathrm{CLSI}^{20}$, pero susceptible para neumonía de acuerdo con los criterios clínicos para neumonía del DRSPWG ${ }^{17}$.

En un caso el hemocultivo fue polimicrobiano habiéndose aislado, junto a $S$. pneumoniae, Klebsiella pneumoniae, en un paciente alcohólico que falleció.

Se detectaron las siguientes complicaciones en 11 pacientes: cuatro ingresaron a UTI y requirieron VM, cuatro requirieron la colocación de un tubo de tórax por efusión pleural complicada (empiema), dos presentaron neumonía de lenta resolución y un caso con infección por VIH diagnosticado durante la interna- ción presentó una disfunción sistólica con dilatación ventricular que revirtió con la mejoría de la neumonía.

Excluyendo los pacientes fallecidos, los derivados a UTI y un caso que permaneció internado 100 días por razones sociales, los pacientes estuvieron internados en promedio ( \pm DS) 8,8 $( \pm 4$ ) días (rango 3-19 días). Fallecieron cuatro pacientes $(8,2 \%)$ durante la internación, tres casos correspondían al grupo $\mathrm{V}$ y uno al grupo IV del ISN.

Todos los pacientes recibieron tratamiento inicial durante la internación por vía parenteral, se utilizaron siete esquemas antimicrobianos diferentes para el tratamiento empírico inicial, los esquemas utilizados y los cambios realizados se muestran en la Figura 1. Considerando los 37 pacientes donde se redujo el espectro
Figura 1. Pautas antimicrobianas utilizadas y secuencia de administración de antibacterianos en pacientes adultos con neumonía bacteriémica causada por Streptococcus pneumoniae.

*En tres pacientes se indicó cotrimoxazol por sospecha de infección por Pneumocystis jirovecii y en un caso metronidazol por dolor abdominal y diarrea.

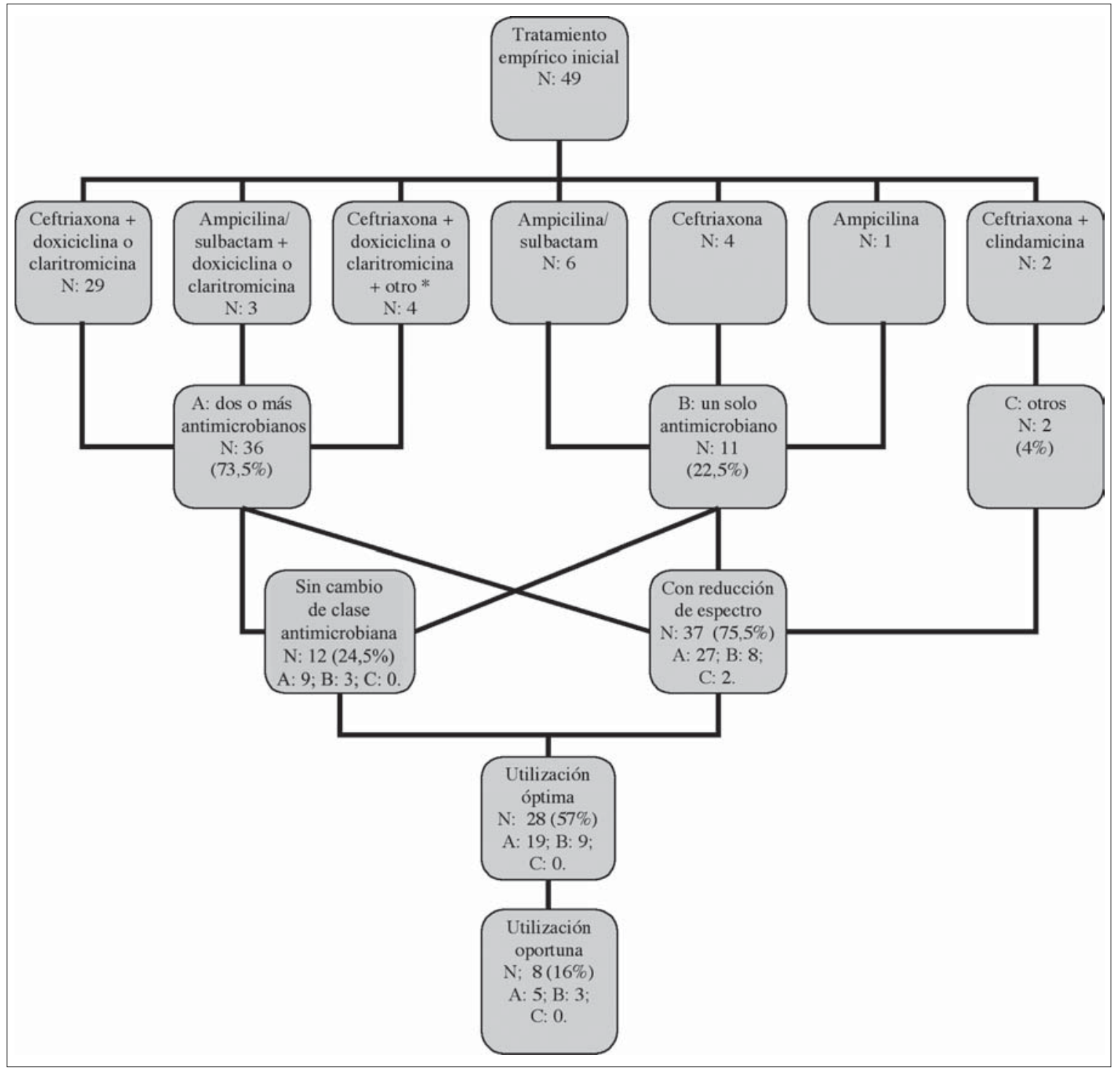


antibacteriano, el cambio se realizó en promedio ( \pm DS) a los 5,9 ( $\pm 1,9)$ días (rango 3-10 días). En los casos donde se realizó la prescripción de penicilina, ampicilina o amoxicilina, se indicó en promedio ( \pm DS) a los 6,2 ( \pm 2) días, (rango 3-10 días). Los motivos por los que no se realizaron cambios en la clase antimicrobiana del tratamiento empírico inicial (n: 12) fueron los siguientes: un paciente recibió tratamiento con ampicilina como único antibacteriano desde el ingreso por lo que no fue necesario modificar el tratamiento por el informe del hemocultivo, en un caso no se modificó por alergia a penicilina, en cinco por alta médica o voluntaria antes de recibir el informe microbiológico completo y en cinco pacientes por complicaciones y mala evolución. De los 10 pacientes en los que se redujo el espectro antibacteriano pero no recibieron como tratamiento definitivo penicilina, ampicilina y/o amoxicilina, con una utilización parcial del informe microbiológico, correspondieron a la suspensión de cotrimoxazol o clindamicina en tres casos, en dos pacientes se cambió ceftriaxona por ampicilina pero continuaron en un caso con doxiciclina sin justificación y con clindamicina en el otro paciente por sospecha de infección con anaerobios por alcoholismo con alteración de la conciencia y neumonía de lenta resolución, en un paciente se suspendió claritromicina pero se continuó el tratamiento con ceftriaxona por hemocultivo positivo para $K$. pneumoniae, en los cuatro casos restantes se suspendió el tratamiento para microorganismos atípicos pero se pasó a vía oral con amoxicilina/ác clavulánico sin tener en cuenta los resultados del hemocultivo. De los 36 pacientes que recibieron inicialmente tratamiento con claritromicina o doxiciclina para cubrir microorganismos atípicos, el mismo fue suspendido en $24(67 \%)$. De los 28 pacientes en los que se realizó una utilización óptima del informe microbiológico, en 12 casos la indicación coincidió con el cambio a vía oral con amoxicilina (en dos de ocho dentro de las primeras 96 horas y en 10 de los 20 restantes en forma tardía), (Tablas 2 y 3 y Figura 1 ).

Comparando los pacientes que no recibieron penicilina o amoxicilina como único antimicrobiano con aquellos que sí lo recibieron y con el objetivo de encontrar una justificación para el no cambio de terapia que pudiera relacionarse con la gravedad de los pacientes y otras variables clínicas y epidemiológicas, se observaron diferencias significativas en la clase V del ISN (23,8 vs 3,6\%; p: 0,04) (Tabla 4), en la presión arterial $\leq 90 / 60 \mathrm{mmHg}(33,3$ vs 3,6\%; p: 0,007), la presencia de complicaciones $(38,1$ vs $10,7 \%$; p: 0,02), derivación a UTI y apoyo con VM (19 vs 0\%; p: 0,02), antibioterapia previa $(23,8$ vs $0 \%$; p: 0,01$)$, presencia de diabetes mellitus $(33,3$ vs $3,6 \%$; p: 0,007$)$ y mortalidad (19 vs $0 \%$; p: 0,02). También se observaron diferencias significativas en la media ( \pm DS) de los días de internación $(7 \pm 3,6$ días $v s$ 9,7 $\pm 3,9$ días; p: 0,03) y de los siguientes valores de laboratorio: albuminemia $(2,8 \pm$ $0,5 \mathrm{~g} / \mathrm{dl}$ vs 3,2 $\pm 0,4 \mathrm{~g} / \mathrm{dl}$; p: 0,01), leucocitos (21.609 \pm $7.497 / \mathrm{mm}^{3}$ vs $\left.17.353 \pm 6.078 / \mathrm{mm}^{3} ; \mathrm{p}: 0,03\right)$ y en $\mathrm{la} \mathrm{PaO}_{2} /$ $\mathrm{FIO}_{2}(288 \pm 60$ vs $379 \pm 139 ; \mathrm{p}: 0,03)$.

No se demostró una asociación significativa entre la no indicación de penicilina o amoxicilina como único antimicrobiano y las siguientes variables analizadas en forma dicotómica: edad $>65$ años, sexo, factores de riesgo, dos o más factores de riesgo, hepatopatía crónica-cirrosis, EPOC, infección por VIH, internaciones previas en el último año, neumonías previas, manifestaciones clínicas (fiebre, tos, expectoración, disnea, dolor torácico, calofríos, cefalea, dolor abdominal, auscultación patológica, vómitos, diarrea, confusión mental, oliguria, shock), frecuencia cardíaca $\geq 100 / \mathrm{min}$, frecuencia respiratoria $\geq 30 / \mathrm{min}, \mathrm{PaO}_{2} / \mathrm{FIO}_{2}<300$, uremia $\geq 45 \mathrm{mg} / \mathrm{dl}$, natremia $\leq 130 \mathrm{mEq} / 1$, creatininemia $\geq 1,5$ $\mathrm{mg} / \mathrm{dl} \mathrm{y} \geq 2 \mathrm{mg} / \mathrm{dl}$, albuminemia $\leq 3 \mathrm{~g} / \mathrm{dl}$, leucocitos $>$

Tabla 2. Relación entre el resultado del hemocultivo y el curso del tratamiento antimicrobiano en pacientes adultos con neumonía bacteriémica causada por Streptococcus pneumoniae

Conducta en relación a esquema antimicrobiano inicial Pacientes

n (\%)

Cambio con reducción de espectro, fármaco menos tóxico

o de menor costo

$(75,5)$

Indicado por hemocultivo

No indicado por hemocultivo

Sin cambio de clase antimicrobiana

Hemocultivo que indica continuar con el tratamiento empírico

Continua el tratamiento empírico a pesar de que el hemocultivo indica

un cambio
33

4

12

1

11
$(67,5)$

(8)

$(24,5)$

(2)

$(22,5)$
Tabla 3. Cambios en el tratamiento antimicrobiano de pacientes adultos con neumonía bacteriémica causada por Streptococcus pneumoniae

Cambio al esquema antimicrobiano inicial Pacientes

n (\%)

Suspensión del macrólido $5 \quad(10)$

Cambio a penicilina y/o amoxicilina*

Cambio a penicilina y/o amoxicilina + suspensión del macrólido

Suspensión de otro antimicrobiano

Sin cambios de clase*

$10 \quad(20,5)$

19 (39)

$3 \quad(6)$

$12(24,5)$

*De los 10 casos que cambiaron a penicilina o amoxicilina, uno continuó el tratamiento con doxiciclina y otro con clindamicina y no fueron considerados tratamientos óptimos de acuerdo al hemocultivo y en el grupo sin cambio de clase antimicrobiana un caso recibió tratamiento con ampicilina desde el ingreso y fue considerado óptimo (total 28 casos). 
Tabla 4. Relación entre el índice de gravedad de neumonía e indicación de tratamiento guiado por el hemocultivo en pacientes adultos con neumonía bacteriémica por Streptococcus pneumoniae

\begin{tabular}{|c|c|c|c|c|c|c|}
\hline ISN & $\begin{array}{c}\text { Número } \\
\mathbf{n}\end{array}$ & $\begin{array}{l}\text { de casos } \\
(\%)\end{array}$ & $\begin{array}{l}\text { Sin cambio de } \\
\text { clase antimicrobiana }\end{array}$ & $\begin{array}{l}\text { Reducción de } \\
\text { espectro }\end{array}$ & $\begin{array}{c}\text { Óptimo } \\
\text { (penicilina, ampicilina, } \\
\text { amoxicilina) }\end{array}$ & $\begin{array}{c}\text { Oportuno } \\
\text { (dentro de las } \\
\text { primeras } 96 \text { horas) }\end{array}$ \\
\hline 1 & 3 & (6) & 1 & 2 & $2 \quad(67 \%)$ & 0 \\
\hline 11 & 9 & $(18,5)$ & 1 & 8 & $6 \quad(67 \%)$ & $1 \quad(11 \%)$ \\
\hline III & 10 & $(20,5)$ & 4 & $6^{*}$ & $6 *(60 \%)$ & $4 \quad(40 \%)$ \\
\hline IV & 21 & (43) & 4 & 17 & $13 \quad(62 \%)$ & $2 \quad(9,5 \%)$ \\
\hline V & 6 & $(12)$ & 2 & 4 & $1 \quad(17 \%)$ & $1 \quad(17 \%)$ \\
\hline Total & 49 & $(100)$ & 12 & 37 & $28 \quad(57 \%)$ & $8 \quad(16 \%)$ \\
\hline
\end{tabular}

$20.000 / \mathrm{mm}^{3}$, hematocrito $\leq 30 \%$, hemoglobina $\leq 10 \mathrm{~g} / \mathrm{dl}$, extensión radiográfica y presencia de efusión pleural y en los promedios de edad, glicemia, hematocrito, hemoglobina, uremia y creatininemia.

\section{Discusión}

Las características epidemiológicas, clínicas, de laboratorio, radiológicas, la gravedad de la neumonía y la mortalidad de nuestra serie son, en general, similares a lo descrito en la literatura científica sobre neumonía neumocóccica bacteriémica, con algunas diferencias menores como la baja frecuencia de insuficiencia cardíaca dentro de las co-morbilidades en esta población ${ }^{21-24}$.

El uso frecuente e indiscriminado de antibacterianos de amplio espectro como las cefalosporinas de tercera generación para el tratamiento de la NAC es uno de los principales factores que contribuyen al problema de la resistencia antimicrobiana a éstas y a otros grupos de antibacterianos, particularmente en los bacilos gramnegativos como Pseudomonas aeruginosa y Acinetobacter sp con cepas productoras de $\beta$-lactamasas de espectro extendido y cepas hiperproductoras de cefalosporinasas $(\mathrm{AmpC})^{25-28}$. La identificación de una cepa de $S$. pneumoniae susceptible en el hemocultivo es una oportunidad para reducir el espectro antibacteriano suspendiendo la cobertura para microorganismos atípicos y cambiando el tratamiento antimicrobiano por penicilina, ampicilina o amoxicilina con al menos la misma efectividad que los antimicrobianos de amplio espectro pero contribuyendo a reducir el problema de la resistencia de $S$. pneumoniae y otras especies a antimicrobianos y con menores costos según el esquema utilizado para el tratamiento empírico inicial de la $\mathrm{NAC}^{9,29}$.
En nuestro estudio, en $75,5 \%$ de los pacientes se modificó el tratamiento empírico inicial con reducción del espectro antibacteriano, en $67 \%$ se suspendió la cobertura para microorganismos atípicos y en $57 \%$ se modificó el tratamiento inicial por penicilina, ampicilina o amoxicilina como único antimicrobiano, con una utilización aceptable del informe microbiológico por parte de los médicos tratantes, comparado con lo antes reportado en la literatura médica. En el estudio de Waterer GW y cols, el hemocultivo positivo para $S$. pneumoniae alteró el manejo de 41,9\% (31/74) de los pacientes, en dos casos fue por resistencia a penicilina. Sólo en 11 pacientes se indicó penicilina $(21,6 \%)$ y se suspendió la cobertura para agentes atípicos en 13/35 casos en los que se indicó este tratamiento $(37,1 \%)^{9}$. En un estudio español se describen cifras aún inferiores, con un cambio del tratamiento inicial en $11 \%$ y una indicación de penicilina en las cepas de $S$. pneumoniae sensibles de $4,1 \%{ }^{21}$. En una serie prospectiva realizada en Chile, con intervención específica de asesoría infectológica, se prescribió penicilina o amoxicilina en 36 de 50 pacientes $(72 \%)$ con neumonía neumocóccica bacteriémica $^{30}$.

Comparando el grupo en el que no se prescribió tratamiento con penicilina o amoxicilina en forma exclusiva con el que sí recibió estos antimicrobianos (utilización óptima del hemocultivo) se encontraron diferencias estadísticamente significativas en la presencia de una presión arterial $\leq 90 / 60$, en el ISN grupo $\mathrm{V}$, la presencia de complicaciones, derivación a UTI con apoyo de VM, mortalidad, antibioticoterapia previa, presencia de diabetes mellitus, mayor valor en promedio de leucocitos y menores valores en promedio de albuminemia y $\mathrm{PaO}_{2} / \mathrm{FIO}_{2}$. Estos hallazgos sugieren que al menos uno de los factores por los que los médicos tratantes no prescriben penicilina a pesar del hemocultivo con $S$. pneumoniae sensible está relacio- 
nado con la gravedad de los pacientes con neumonía neumocóccica. Otras variables relacionadas con la gravedad de la NAC como la confusión mental, el compromiso multilobar o la uremia elevada es probable que no tuvieran diferencias significativas por el número de pacientes incluidos en este trabajo. Estudios retrospectivos avalan esta conducta refiriendo que la terapia combinada, principalmente un antibacteriano $\beta$ lactámico con un macrólido, mostró menor riesgo de mortalidad en aquellos enfermos con NAC bacteriémica por $S$. pneumoniae que fueron tratados con la combinación mencionada ${ }^{31-33}$. Un estudio prospectivo reciente no mostró diferencias significativas, en general, entre terapia combinada y monoterapia en el tratamiento de la NAC bacteriémica por $S$. pneumoniae. Sin embargo, cuando los autores analizaron el subgrupo de los pacientes con NAC grave, hallaron diferencias significativas en la mortalidad a los 14 días entre los enfermos que se trataron con terapia combinada y con monoterapia $(23,4$ vs $55,3 \%)$, sin que dicha diferencia fuera atribuible a ningún fármaco en particular ${ }^{34}$. Debido a los diferentes esquemas antimicrobianos utilizados y la baja mortalidad hospitalaria, no es posible relacionar los diferentes tratamientos y sus modificaciones con el pronóstico de los pacientes en este trabajo. Otra causa en nuestra serie fue la llegada del informe microbiológico luego del alta hospitalaria del paciente, también reportado en publicaciones previas ${ }^{9,21}$ y coincidente con el hallazgo de una menor estancia hospitalaria, en promedio, que aquellos que cambiaron por penicilina o amoxicilina como único antimicrobiano. Otros factores citados en la literatura científica que pueden influir en la conducta médica de no indicar el tratamiento antimicrobiano basado en el antibiograma son la posibilidad de una etiología múltiple de la neumonía en un porcentaje apreciable de los $\operatorname{casos}^{35}$, siendo la asociación entre Mycoplasma pneumoniae y $S$. pneumoniae una de las más frecuentes y la comodidad posológica de algunos antibacterianos de amplio espectro (1-2 veces por día) por sus propiedades farmacocinéticas, y fármaco-económicas en comparación con las múltiples dosis diarias que se requieren de penicili$\mathrm{na}^{21,30}$. Otro causal reportado es el temor a problemas médico/legales por parte de los médicos tratantes ${ }^{9}$.

Un aporte de este trabajo con respecto a la conducta médica cuando en los pacientes con NAC se aísla en los hemocultivos $S$. pneumoniae, es el hallazgo de una excesiva demora en realizar los cambios, ya que sólo en ocho pacientes $(16 \%)$ se indicó penicilina dentro de las primeras 96 horas de internación, cuando el informe microbiológico puede estar disponible en 48 a 72 horas. Esta variable no ha sido comunicada en estudios previos sobre la utilidad de los hemocultivos en la neumonía neumocóccica bacteriémica. Una limitación de nuestro estudio es el diseño retrospectivo que no permite definir con certeza la causa de la demora, pudiendo contribuir por un lado el retraso en el informe microbiológico completo y por el otro una conducta expectante del médico tratante ante la evolución favorable del paciente con el tratamiento empírico inicial. Otro dato de interés es que en $50 \%$ de los casos con cambio tardío, el mismo coincidió con el paso a vía oral del tratamiento antimicrobiano permitiendo suponer que el informe microbiológico sólo fue tenido en cuenta por el médico tratante en esta instancia del tratamiento, con el agravante de que el pasaje de la vía endovenosa a la vía oral es una de las recomendaciones de las guías de práctica clínica que tiene un bajo cumplimiento por parte de los médicos tratantes en Argentina $^{36}$.

De acuerdo con el criterio poblacional de resistencia a penicilina de $S$. pneumoniae del CLSI año $2008^{20}$, sólo un caso presentó resistencia intermedia a penicilina, cifras inferiores a las reportadas en nuestro medio para la población pediátrica ${ }^{37}$, pero coincidente con estudios realizadas sobre bacteriemias neumocóccicas en pacientes adultos de Argentina, donde el porcentaje de cepas invasoras con SDP varía entre 0 y 4,6\%. En la mayoría de los estudios nacionales se demuestra que las cepas aisladas de $S$. pneumoniae en la población adulta son susceptibles a penicilina, a diferencia de lo que ocurre con la población pediátrica ${ }^{24,38,39}$. Utilizando como punto de corte los criterios clínicos propuestos por el DRSPWG ${ }^{17}$ para neumonía, todas las cepas fueron sensibles a penicilina, por lo que la posible resistencia del $S$. pneumoniae no debiera ser una causa de tratamiento con ceftriaxona o quinolonas en pacientes adultos con NAC de nuestro medio, a diferencia de lo propuesto en algunas guías de práctica clínica en otros países ${ }^{4,5}$.

Debido a los beneficios y ventajas potenciales de penicilina en mejorar el problema de la resistencia antimicrobiana, y en algunos casos reducir los costos del tratamiento de la neumonía neumocóccica bacteriémica, es importante trabajar sobre las prácticas de prescripción de los médicos clínicos, no sólo en términos de reducción del espectro antibacteriano y la indicación de penicilina sino también al manejo de los tiempos adecuados para la indicación del cambio, mejorando la coordinación y el trabajo en equipo con el laboratorio de microbiología.

\section{Resumen}

Evaluamos la utilidad de los hemocultivos en el manejo terapéutico de pacientes con neumonía neumocóccica bacteriémica internados en un servicio de clínica médica. Estudio observacional, retrospectivo. 
Se incluyeron 49 pacientes, $75,5 \%$ hombres, edad media 51,1 años. Todas las cepas de $S$. pneumoniae fueron susceptibles a penicilina. Fallecieron 4 pacientes. En $75,5 \%$ se modificó el tratamiento con reducción del espectro antibacteriano, en $57 \%$ se cambió a bencilpenicilina o amoxicilina como único antimicrobiano, pero sólo en $16 \%$ se realizó dentro de los primeros cuatro días. En 12 casos la indicación coinci- dió con el cambio a vía oral por amoxicilina. Debido a los beneficios y ventajas potenciales de penicilina en mejorar el problema de la resistencia antimicrobiana, y en algunos casos reducir los costos, es importante trabajar sobre las prácticas de prescripción de los médicos clínicos, acerca de la indicación de penicilina y al momento oportuno del cambio, mejorando la utilización del informe microbiológico.

\section{Referencias}

1.- Bantar C, Bavestrello L, Curcio D, Jasovich A, Absi R, Bagnulo H, et al. Acute community-acquired pneumonia in adults: guidelines for initial antimicrobial therapy based on local evidence from a South American Working Group (ConsenSur). J Chemother 2002; 14 (Suppl 4): 1-22.

2.- Luna C M, Calmaggi A, Caberloto O, Gentile J, Valentini R, Ciruzzi J, et al. Neumonía adquirida en la comunidad. Guía práctica elaborada por un comité intersociedades. Medicina (B Aires) 2003; 63: 319-43.

3.- Bartlett J G, Dowell S F, Mandell L A, File Jr T M, Musher D M, Fine M J. Practice guidelines for the management of community-acquired pneumonia in adults. Infectious Diseases Society of America. Clin Infect Dis 2000; 31: 347-82.

4.- Mandell L A, Bartlett J G, Dowell S F, File Jr T M, Musher D M, Whitney C. Update of practice guidelines for the management of community-acquired pneumonia in immunocompetent adults. Clin Infect Dis 2003; 37: 1405-33.

5.- Niederman M S, Mandell L A, Anzueto A, Bass J B, Broughton W A, Campbell G D, et al. Guidelines for management of adults with community-acquired pneumonia. Am J Resp Crit Care Med 2001; 163 : 1730-54.

6.- British Thoracic Society. Guidelines for the Management of Community Acquired Pneumonia in Adults. Thorax 2001; 56 (Suppl 4): 1-64.

7.- Díaz A, Calvo M, O'Brien A, Farías G, Mardónez J M, Saldías F. Clinical usefulness of blood cultures in hospitalized patients with community-acquired pneumonia. Rev Méd Chile 2002; 130: 993-1000.

8.- Campbell S G, Marrie T J, Anstey R, Dickinson G, Ackroyd-Stolarz S. The contribution of blood cultures to the clinical management of adult patients admitted to the hospital with community-acquired pneumonia. A prospective observational study. Chest 2003; 123: 1142-50.
9.- Waterer G W, Jennigs S G, Wunderink R G. The impact of blood cultures on antibiotic therapy in pneumococcal pneumonia. Chest 1999; 116: 1278-81.

10.- Musher D M, Alexandraki I, Graviss E A, Yanbeiy N, Eid A, Inderias LA, et al. Bacteremic and nonbacteremic pneumococcal pneumonia. A prospective study. Medicine (Baltimore) 2000; 79: 210-21.

11.- Napolitano L, Swartz M. Neumonía adquirida en la comunidad. Lee B, Hsu S, Stasior D, editors. Medicina basada en la evidencia Massachusetts General Hospital. Edición en español. Madrid: Marban, S.L; 1999, p. 577605

12.- Lippmann M L, Goldberg S K, Walkenstein M D, Herring W, Gordon M. Bacteremic pneumococcal pneumonia. A community hospital experience. Chest 1995; 108: 1608-13.

13.- Appelbaum P C. Antimicrobial resistance in streptococcus pneumoniae: an overview. Clin Infect Dis 1992; 15: 77-83.

14.- Trampuz A, Widmer A F, Fluckiger U, Haenggi M, Frei R, Zimmerli W. Changes in the epidemiology of pneumococcal bacteremia in a Swiss university hospital during a 15-year period, 1986-2000. Mayo Clin Proc 2004; 79: 604-12.

15.- Vallès X, Marcos A, Pinart M, Piñer R, Marco F, Mensa J M, et al. Hospitalized community-acquired pneumonia due to Streptococcus pneumoniae: Has resistance to antibiotics decreased? Chest. 2006; 130: 800-6

16.- Ewig S, Ruiz M, Torres A, Marco F, Martínez J A, Sánchez M, et al. Pneumonia acquired in the community through drugresistant Streptococcus pneumoniae. Am J Respir Crit Care Med. 1999; 159: 1835-42

17.- Heffelfinger J D, Dowell S F, Jorgensen J H, Klugman K P, Mabry L R, Musher D M, et al, Management of community-acquired pneumonia in the era of pneumococcal resistance. A report of The Drug-Resistant Streptococcus pneumoniae Therapeutic Working Group. Arch Intern Med 2000; 160: 1399-408.
18.- Fine M, Auble T E, Yealy D M, Hanusa B H, Wiessfeld L A, Singer D E, et al. A prediction rule to identify low risk patients with community acquired pneumonia. N Engl J Med 1997; 336: 243-50.

19.- Clinical and Laboratory Standards Institute. Performance standards for antimicrobial disk susceptibility tests. Ninth edition. Approved Standard. CLSI document M2-A9. Clinical and Laboratory Standards Institute, Wayne, $\mathrm{Pa}$, USA, 2006.

20.- Clinical and Laboratory Standards Institute Performance standards for Antimicrobial susceptibility testing. Eighteenth informational supplement. M100-S18. CLSI, Wayne, PA. USA, 2008.

21.- Núñez Fernández M J, Ojea de Castro R, Lueiro Lores F, Pulian Morais MV, Hernández Blanco $M$, Núñez Fernández $M$, et al. Neumonía neumocócica con bacteriemia en adultos: estudio descriptivo en el noroeste de España. An Med Interna (Madrid) 2002; 19: 612-20

22.- Rioseco M L, Riquelme R. Neumonía neumocócica bacterémica en 45 adultos inmunocompetentes hospitalizados. Cuadro clínico y factores pronósticos. Rev Méd Chile 2004; 132: 588-94.

23.- Shariatzadeh M R, Huang J Q, Tyrrell G J, Johnson M M, Marrie T J. Bacteremic pneumococcal pneumonia: a prospective study in Edmonton and neighboring municipalities. Medicine (Baltimore) 2005; 84: $147-61$

24.- Gentile J H, Sparo M D, Mercapide M E, Luna C M. Adult bacteremic pneumococcal pneumonia acquired in the community. A prospective study on 101 patients. Medicina (B Aires). 2003; 63: 9-14.

25.- Hanson N D. AmpC beta-lactamases: what do we need to know for the future? Antimicrob Chemother 2003; 52: 2-4.

26.- Patterson J E. Extended-spectrum betalactamases. Sem Res Crit Care Med 2003; 24: 79-87.

27.- Gold H S, Moellering R C. Antmicrobial-drug resistance. N Engl J Med 1996; 335: 1445-53.

28.- Dancer S J. The problem with 
cephalosporins. J Antimicrob Chemother 2001; 48: 463-78.

29.- Mella S, Blamey R, Riedel G, Aylwin M, Chabouty H. Utilidad de penicilina $\mathrm{G}$ en el tratamiento de la neumonía neumocócica. Rev Chil Infect 2005; 22: 377-8.

30.- Mella S, Blamey R, Paiva O, Yáñez J, Riedel G, Aylwin M, et al. Impacto de la evaluación infectológica en el uso racional de antimicrobianos en pacientes con bacteriemia por Streptococcus pneumoniae. Rev Chil Infect 2007; 24: 264-9

31.- File Jr T M, Mandell L A. What is optimal antimicrobial therapy for bacteremic pneumococcal pneumonia?. Clin Infect Dis 2003; 36: 396-8.

32.- Martínez J A, Horcajada J P, Almela M, Marco F, Soriano A, García E, et al. Addition of a macrolide to a $\beta$-lactam based empirical antibiotic regimen is associated with lower in-hospital mortality for patients with bacteremic pneumococcal pneumonia. Clin Infect Dis 2003; 36: 389-95.

33.- Waterer GW, Somes GW, Wunderink R G. Monotherapy may be suboptimal for severe bacteremic pneumococcal pneumonia. Arch Intern Med 2001; 161: 1837-42.

34.- Baddour LM, Yu VL, Klugman KP, Feldman C, Ortqvist A, Rello J, et al. Combination antibiotic therapy lowers mortality among severely ill patients with pneumococcal bacteremia. Am J Respir Crit Care Med 2004; 170: 440-4.

35.- Lieberman D, Schlaefer F, Boldur I. Multiple pathogens in adult patients admitted with community-acquired pneumonia: a one year prospective study of 346 consecutive patients. Thorax 1996; 51: 179-84.

36.- Christensen D, Luna CM, Martínez J, Rodríguez E, Marzoratti L, González J, et al. Cumplimiento con las guías nacionales en pacientes hospitalizados con neumonía adquirida en la comunidad.
Resultados del estudio CAPO en Argentina. Medicina (Buenos Aires) 2007; 67: 709-13.

37.- Ruvinsky R. Epidemiología de las infecciones invasoras por Streptococcus pneumoniae en la Argentina: un sistema de vigilancia, 1993-2004. Rev Hosp Niños B Aires 2005; 47: 222-32.

38. - Radice M, Kovensky J, Pasterán F. Perfil de sensibilidad de diferentes antimicrobianos sobre Streptococcus pneumoniae aislados de pacientes adultos con infecciones no meníngeas. XVII Congreso Latinoamericano de Microbiología. Buenos Aires, Argentina 2004. Res A-44.

39.- Acosta-Gnass S, Benchetrit G, Bermejo J, Crámaro $\mathrm{H}$, Ezcurra $\mathrm{C}$, Gentile $\mathrm{J}$, et al. Neumonía neumocóccica bacteriémica (NNB): estudio multicéntrico de factores pronósticos. VI Congreso Argentino SADI 2006. Mar del Plata, Argentina 2006. Res 263. 